University of Wollongong

Research Online

Faculty of Engineering and Information

Faculty of Engineering and Information

Sciences - Papers: Part A

Sciences

$1-1-2015$

\title{
Molecular dynamics study on the grain boundary dislocation source in nanocrystalline copper under tensile loading
}

\author{
Liang Zhang \\ University of Wollongong, Iz592@uowmail.edu.au \\ Cheng Lu \\ University of Wollongong, chenglu@uow.edu.au \\ A Kiet Tieu \\ University of Wollongong, ktieu@uow.edu.au \\ Linqing Pei \\ University of Wollongong, Ip115@uowmail.edu.au \\ Xing Zhao \\ University of Wollongong, xz920@uowmail.edu.au
}

See next page for additional authors

Follow this and additional works at: https://ro.uow.edu.au/eispapers

Part of the Engineering Commons, and the Science and Technology Studies Commons

Research Online is the open access institutional repository for the University of Wollongong. For further information contact the UOW Library: research-pubs@uow.edu.au 


\title{
Molecular dynamics study on the grain boundary dislocation source in nanocrystalline copper under tensile loading
}

\author{
Abstract \\ Grain boundary (GB) is the interface between different oriented crystals of the same material, and it can \\ have a significant effect on the many properties of materials. When the average or entire range of grain \\ size is reduced to less than $100 \mathrm{~nm}$, the conventional plastic deformation mechanisms dominated by \\ dislocation processes become difficult and GBmediated deformation mechanisms become increasingly \\ important. One of the mechanisms that can play a profound role in the strength and plasticity of metallic \\ polycrystalline materials is the heterogeneous nucleation and emission of dislocations from $\mathrm{GB}$. In this \\ study, we conducted molecular dynamics simulations to study the dislocation nucleation from copper \\ bicrystal with a number of $\langle 110\rangle$ tilt GBs that covered a wide range of misorientation angles $(\theta)$.Wewill \\ show from this analysis that the mechanic behavior of GBs and the energy barrier of dislocation \\ nucleation from GBs are closely related to the lattice crystallographic orientation, GBenergy, and the \\ intrinsic GBstructures. An atomistic analysis of the nucleation mechanisms provided details of this \\ nucleation and emission process that can help us to better understand the dislocation source in GB.
}

\section{Keywords}

tensile, molecular, dynamics, study, loading, grain, under, boundary, dislocation, source, nanocrystalline, copper

\section{Disciplines}

Engineering | Science and Technology Studies

\section{Publication Details}

Zhang, L., Lu, C., Tieu, K., Pei, L., Zhao, X. \& Cheng, K. (2015). Molecular dynamics study on the grain boundary dislocation source in nanocrystalline copper under tensile loading. Materials Research Express, 2 (3), 035009-1-035009-14.

\section{Authors}

Liang Zhang, Cheng Lu, A Kiet Tieu, Linqing Pei, Xing Zhao, and Kuiyu Cheng

This journal article is available at Research Online: https://ro.uow.edu.au/eispapers/3982 


\title{
Molecular dynamics study on the grain boundary dislocation source in nanocrystalline copper under tensile loading
}

\author{
Liang Zhang, Cheng Lu*, Kiet Tieu, Linqing Pei, Xing Zhao, Kuiyu Cheng \\ School of Mechanical, Materials and Mechatronic Engineering, University of Wollongong, Wollongong, \\ NSW 2522, Australia
}

*Corresponding author: chenglu@uow.edu.au (C. Lu)

\begin{abstract}
Grain boundary (GB) is the interface between different oriented crystals of the same material, and it can have a significant effect on the many properties of materials. When the average or entire range of grain size is reduced to less than $100 \mathrm{~nm}$, the conventional plastic deformation mechanisms dominated by dislocation processes become difficult and GB mediated deformation mechanisms become increasingly important. One of the mechanisms that can play a profound role in the strength and plasticity of metallic polycrystalline materials is the heterogeneous nucleation and emission of dislocations from GB. In this study, we conducted molecular dynamics simulations to study the dislocation nucleation from copper bicrystal with a number of $\langle 110\rangle$ tilt GBs that covered a wide range of misorientation angles $(\theta)$. We will show from this analysis that the mechanic behavior of GBs and the energy barrier of dislocation nucleation from GBs are closely related to the lattice crystallographic orientation, GB energy, and the intrinsic GB structures. An atomistic analysis of the nucleation mechanisms provided details of this nucleation and emission process that can help us to better understand the dislocation source in GB.
\end{abstract}

Keywords: molecular dynamics; grain boundary; dislocation nucleation; tensile strength

\section{Introduction}

Grain boundary (GB) strengthening at low temperatures is a well known phenomenon in polycrystalline materials. One of the best known theories, described by the Hall-Petch equation, predicts an increase of flow stress with decreasing grain size, but as the grain sizes are reduced to nanometer scale and the percentage of GB atoms correspondingly increases, this traditional view of dislocation-driven plasticity in polycrystalline materials needs to be reconsidered ${ }^{1}$. Experimental measurements ${ }^{2,3}$ have shown that various deviations from the Hall-Petch equations as grain sizes reached nanometer scale. A lot of prior research work conducted by experiments ${ }^{4-7}$ and computational simulations ${ }^{8-13}$ revealed that GBs and their underlying structure can play an important role in the bulk properties of polycrystalline materials, but below a certain critical grain size, in the order of $10 \mathrm{~nm}$, lattice dislocation nucleation becomes limited and GB-mediated processes (e.g., GB sliding, GB migration and grain rotation) becomes the dominant deformation mechanisms ${ }^{8,10}$. While the deformation mechanisms at larger scales have been studied for decades, an atomic level understanding of the GB accommodation mechanism is limited, so most of the recent scientific interest in nanocrystalline materials is associated with the atomic level mechanisms of plastic deformation in the GBs.

As the grain size decreases, one of the mechanisms that can play a vital role in the mechanical behavior of nanocrystalline materials is the heterogeneous nucleation and emission of dislocations from the GBs. This deformation mechanism was obvious in many simulation works ${ }^{14-19}$, and it was also confirmed by in situ transmission electron microscopy (TEM) experiments ${ }^{20,21}$ which showed GBs emitting partial dislocations that formed stacking faults and deformation twins in nanocrystalline $\mathrm{Al}$ and $\mathrm{Cu}$. However, experiments at a nanoscale can be very time consuming and costly, and TEM requires samples with a thickness comparable to the grain size, which may induce the structure to relax and thus change the GB structure ${ }^{1}$, so they are very difficult to perform. Fortunately, molecular dynamics (MD) simulations with a carefully designed model system can be used to investigate GB structures and the dislocation nucleation mechanisms of nanocrystalline materials.

MD simulations were used in previous researches to investigate the nucleation and propagation of dislocations in polycrystal and bicrystal configurations and the results have been fruitful ${ }^{22,23}$. Atomistic studies have shown that differences 
in the mechanical behavior and the underlying deformation mechanisms commonly result from variations in the grain boundary structure ${ }^{24}$. However, the atomic level details of how dislocation nucleation occurs at the grain boundary and what is the correlation of GB structures, GB properties, and the deformation mechanisms of GBs are still not understood very well. Moreover, a characterization of $900 \mathrm{GBs}$ in pure annealed Ni performed by Randle ${ }^{25}$ showed that over half of the interfaces consisted of $\langle 110\rangle$ tilt GBs; this indicated that the $\langle 110\rangle$ tilt GBs are a preferred interface configuration and of importance for face-centered cubic (fcc) materials. We therefore concentrated on the dislocation nucleation from $\langle 110\rangle$ tilt GBs in this study and investigated their underlying atomistic mechanisms. A simulation model of Cu bicrystal was used in this study because the GB geometry and structure can be specified precisely. The GBs that were investigated covered a wide range of misorientation angles $(\theta)$ around the $<110>$ tilt axis.

\section{Methodology}

\subsection{Simulation Model}

The simulations were performed with the parallel molecular dynamics (MD) code LAMMPS ${ }^{26}$. The embedded-atom method (EAM) potential developed by Mishin et al for $\mathrm{Cu}^{27}$ was used because it can fit a large set of experimental and first-principles data. In this study, a bicrystal model was created by constructing two separate grains (grain-A and grain-B in Fig.1-a) with different crystallographic orientation and joined them together along the $\mathrm{Y}$ axis. All the Cu bicrystal models used in this study have a symmetric tilt misorientation about $\left[\begin{array}{lll}1 & \overline{1} & 0\end{array}\right]$ in the direction of the $\mathrm{Z}$ axis. Periodic boundary conditions in the simulation models were applied in all directions ( $\mathrm{X}, \mathrm{Y}$ and $\mathrm{Z}$ axis). Specifically, the periodic boundary conditions were applied in the $\mathrm{X}$ and $\mathrm{Z}$ directions to simulate an infinite boundary plane between the two grains to eliminate the effect of a free surface. It must be noted that the periodic boundary condition in the $\mathrm{Y}$ direction introduced a second boundary plane into the model. A schematic of the bicrystal simulation model is shown in Fig.1(a), and a typical simulation model construction is shown in Fig.1(b). GBs with different rotation angles $(\theta)$ investigated in this study were listed in Table.1. Each bicrystal model was approximately $162 \AA \times 290 \AA \times 162 \AA(X \times Y \times Z)$, and the total number of atoms in each simulation model was approximately $6.5 \times 10^{5}$. Previous work ${ }^{17}$ showed that this cell size is sufficient to avoid image effects from the periodic boundaries on the mechanisms associated with three dimensional dislocation nucleation.

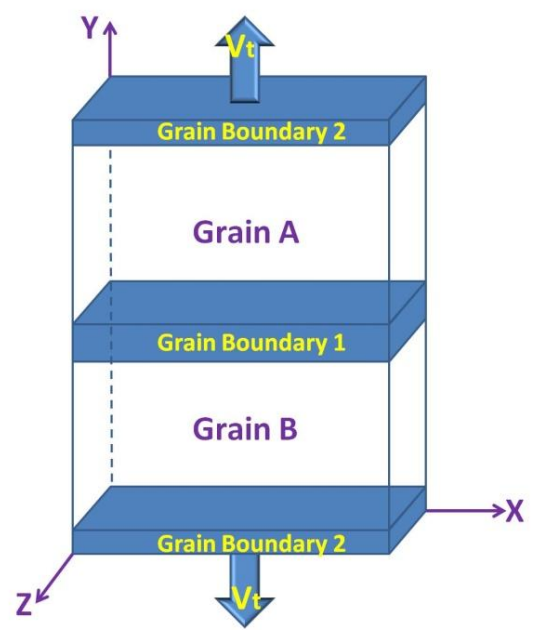

(a)

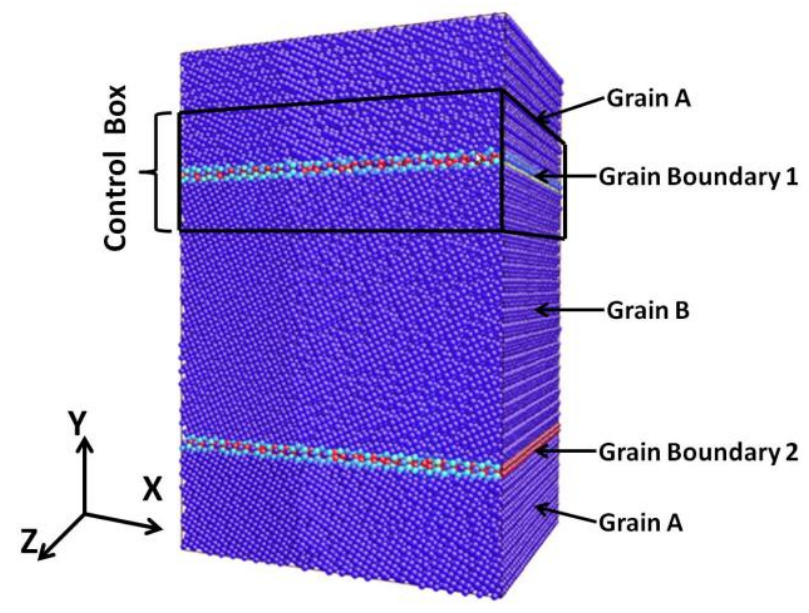

(b)

Figure-1. (a) Schematic of the bicrystal model and the tension deformation applied onto the model. The grain boundary was created by a symmetric tilt rotation of grain-A and grain-B around the [1 $\overline{1} 0]$ direction (z axis). (b) A typical simulation model constructed by LAMMPS and visualized in Atomeye. The atoms were colored according to their potential energy; blue atoms have a perfect fcc structure while the atoms with different colours defined the grain boundary plane.

\subsection{Simulation details}

The equilibrium GB structures were prepared using a combination of molecular statics and MD simulations, that is, a molecular statics calculation with a standard conjugate gradient method was used to determine the minimum energy configurations. A number of initial "starting positions" of grain-A and grain-B were tested to find the best GB structures ${ }^{28-30}$ from the point of view of energy. It is worth noting that for each initial configuration, the size of the model should be 
adjusted to construct identical atomic structures of the two boundary planes. This operation is necessary to ensure they have the same equilibrium structures after their energy has been minimized, otherwise different GB structures in a bicrystal model that have different energies will have a detrimental effect on further simulation results. After attaining minimum energy configuration, the simulation model was equilibrated using MD in the isobaric-isothermal (NPT) ensemble at a pressure of 0 bar and a temperature of $10 \mathrm{~K}$ for $20 \mathrm{ps}$.

During dynamic loading, uniaxial tension at a constant rate of $5 \times 10^{8} / \mathrm{s}$ was applied perpendicular to the boundary plane (along the Y direction), while pressure in the lateral directions was kept at zero. A high strain rate was inherent in the simulations for computational efficiency, and to have the desired amount of deformation within a given simulation time. An isobaric-isothermal (NPT) ensemble was used during the dynamic deformation. The simulation temperature was maintained at $10 \mathrm{~K}$ and an integration time step of $1 \mathrm{fs}$ was used throughout the MD simulations. The fluctuating internal energy of the monitored atoms due to entropy was found to be very small (in the order of $0.0011 \mathrm{eV}$ ) at this temperature ${ }^{31}$, and this low temperature environment actually facilitated our visualisation and analysis of the results. Our focus was on the nucleation mechanisms from the diversity of GBs presented in this study, so the influence of the strain rate and temperature on the results will not be discussed. The visualisation tools Atomeye $\mathrm{e}^{32}$ and Ovito ${ }^{33}$ were used to illustrate the bicrystal models. The common neighbor analysis (CNA) technique ${ }^{34}$ was used to identify the structural defects and their evolution during the simulations. The dislocation extraction algorithm (DXA) ${ }^{35,36}$ was used to compute Burgers vectors of the nucleated dislocations from the boundary plane.

Table.1 Summary information of different $[1 \overline{1} 0]$ symmetric tilt grain boundaries in Cu bicrystal

\begin{tabular}{|c|c|c|c|c|}
\hline $\begin{array}{c}\text { GB plane } \\
\sum(\mathbf{h} \mathbf{k} \mathbf{l}) \\
\end{array}$ & $\begin{array}{c}\text { Misorientation } \\
\text { angel } \theta\left(^{\circ}\right) \\
\end{array}$ & $\begin{array}{c}\text { GB energy } \\
\gamma_{G B}\left(\mathbf{m J} / \mathbf{m}^{2}\right) \\
\end{array}$ & $\begin{array}{c}\text { Tensile strength } \\
\sigma(\mathbf{G P a}) \\
\end{array}$ & $\begin{array}{c}\text { Nucleation barrier } \\
\gamma_{\text {barrier }}\left(\mathbf{m} \mathbf{J} / \mathbf{m}^{3}\right)\end{array}$ \\
\hline$\sum 19\left\{\begin{array}{lll}1 & 1 & 6\end{array}\right\}$ & $26.5^{\circ}$ & 788 & $6.45 / 8.8^{*}$ & 2.23 \\
\hline$\sum 9\left\{\begin{array}{lll}1 & 1 & 4\end{array}\right\}$ & $38.9^{\circ}$ & 684 & 8.94 / 9.41 & 4.13 \\
\hline$\sum 139\{3311\}$ & $42.2^{\circ}$ & 643 & $7.83 / 10.14$ & 3.12 \\
\hline$\sum 11\left\{\begin{array}{lll}1 & 1 & 3\end{array}\right\}$ & $50.5^{\circ}$ & 319 & $9.55 / 10.1$ & 4.85 \\
\hline$\sum 33\{225\}$ & $58.9^{\circ}$ & 581 & $7.97 / 11.16$ & 2.8 \\
\hline$\sum 3\left\{\begin{array}{lll}1 & 1 & 2\end{array}\right\}$ & $70.5^{\circ}$ & 591 & $9.47 / 12.41$ & 4.7 \\
\hline$\sum 17\left\{\begin{array}{lll}2 & 23\}\end{array}\right.$ & $86.6^{\circ}$ & 660 & $9.86 / 14.36$ & 3.35 \\
\hline$\sum 17\left\{\begin{array}{lll}3 & 34\end{array}\right\}$ & $93.4^{\circ}$ & 644 & $11.57 / 14.88$ & 5.05 \\
\hline$\sum 3\left\{\begin{array}{lll}1 & 1 & 1\end{array}\right\}$ & $109.5^{\circ}$ & 26 & $17 / 17$ & - \\
\hline$\sum 171\left\{\begin{array}{lll}11 & 11 & 10\end{array}\right\}$ & $114.5^{\circ}$ & 296 & $1.6 / 13.9$ & 0.1 \\
\hline$\sum 11\left\{\begin{array}{lll}3 & 3 & 2\end{array}\right\}$ & $129.5^{\circ}$ & 535 & $2.57 / 13.48$ & 0.45 \\
\hline$\sum 291\left\{\begin{array}{lll}11 & 11 & 7\end{array}\right\}$ & $131.5^{\circ}$ & 810 & $2.86 / 12.77$ & 0.33 \\
\hline$\sum 9\left\{\begin{array}{lll}2 & 2 & 1\end{array}\right\}$ & $141.1^{\circ}$ & 833 & $4.07 / 11.78$ & 0.75 \\
\hline$\sum 19\left\{\begin{array}{lll}3 & 3 & 1\end{array}\right\}$ & $153.5^{\circ}$ & 856 & $3.87 / 8.82$ & 0.79 \\
\hline$\sum 73\left\{\begin{array}{lll}6 & 61\}\end{array}\right.$ & $166.6^{\circ}$ & 681 & $3.32 / 6.59$ & 0.65 \\
\hline
\end{tabular}

* The maximum tensile stress of single crystal are listed for comparison.

\subsection{Energy calculation}

For calculating the GB energy and measure the energy barrier for dislocation nucleation from a GB, a control box was placed along the entire grain boundary ${ }^{31}$ (see in Fig.1-b). The energy associated with the GB $\left(\gamma_{\mathrm{GB}}\right)$ is calculated by equation (1), where $\mathrm{E}_{\text {box_m }}$ is the potential energy of the control box within the bicrystal model after an energy minimization procedure, $\mathrm{E}_{\text {atom }}$ is the potential energy of a single atom in the perfect $\mathrm{Cu}$ lattice $(-3.54 \mathrm{eV}), \mathrm{N}$ is the total number of atoms contained in the model, and $\mathrm{A}$ is the area of the GB plane $\left(\mathrm{A}=\mathrm{L}_{\mathrm{x}}{ }^{*} \mathrm{~L}_{\mathrm{z}}\right)$. The energy of dislocation nucleation barrier is calculated by equation (2), where $\mathrm{E}_{\text {box_e }}$ is the energy of the control box after the system was equilibrated at $10 \mathrm{~K}$. For each atom $\mathrm{i}$ within the control box, the real-time energy during dynamic tension $\mathrm{E}_{\text {box_t }}^{i}$ was measured at a certain interval 
timestep. $\mathrm{V}$ is the volume of the control box $\left(\mathrm{V}=\mathrm{A} * \mathrm{~L}_{\text {box_y }_{\mathrm{y}}}\right)$, where $\mathrm{L}_{\text {box_y }}$ is the height of the control box.

$\gamma_{\mathrm{GB}}=\frac{\mathrm{E}_{\text {box_m }}-\mathrm{N} \cdot \mathrm{E}_{\text {atom }}}{\mathrm{A}}$

$\gamma_{\text {barrier }}=\frac{\sum_{\mathrm{i}}^{\mathrm{n}} \mathrm{E}_{\text {box_t }}^{\mathrm{i}}-\mathrm{E}_{\text {box_e }}}{\mathrm{V}}$

The size of the control box was fully considered in this work. For example, Fig.2 gives the calculated results of $\sum 9\left(\begin{array}{lll}1 & 1 & 4\end{array}\right)$ GB and $\sum 19$ (3 3 1) GB by using different size control boxes, and where $L_{\text {box }-y}=30 \AA, 60 \AA$ and $200 \AA$. The results showed the same trend and the peak values showed a very small deviation. So the size of the control box had a very small part in the simulation results and would not affect our conclusions. In this work, the value of $\mathrm{L}_{\text {box } y}$ will be set as $60 \AA$ for the calculation, and the results are listed in Table.1.
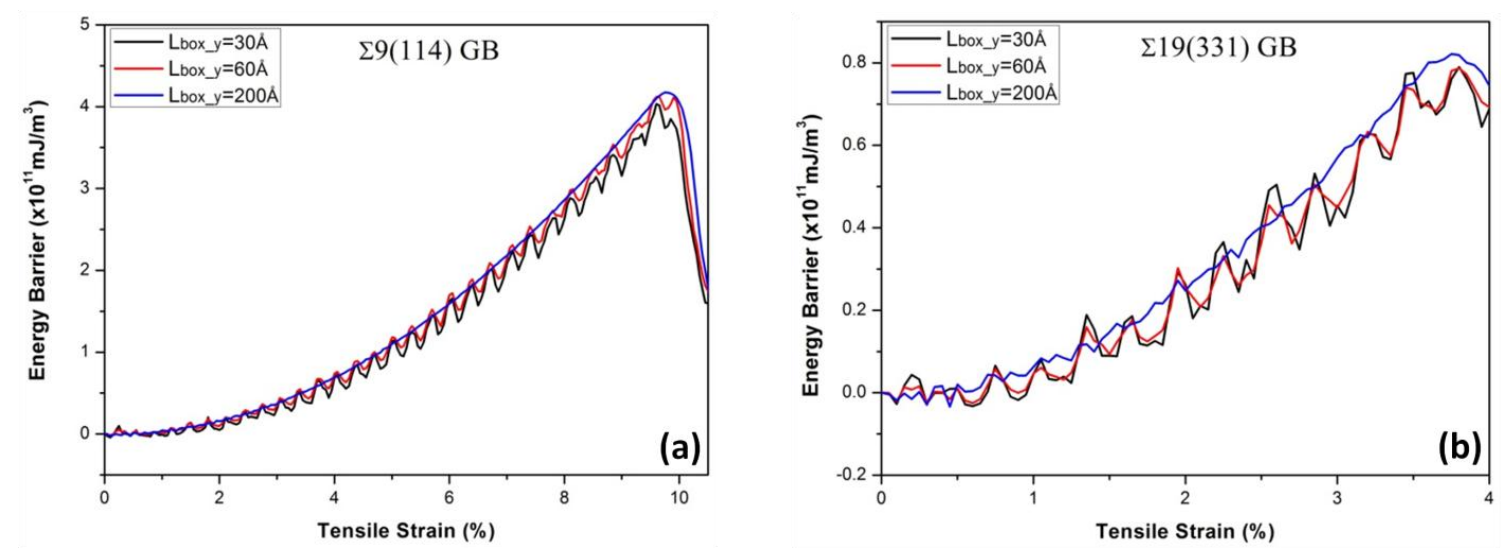

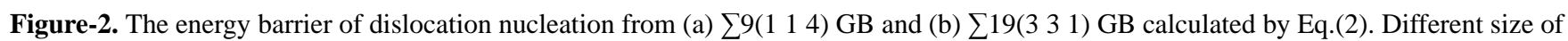
control box with $\mathrm{L}_{\text {box_y }}=30 \AA$ А $60 \AA$ and $200 \AA$ i were tested.

\section{Results and discussions}

\subsection{GB structure and energy}

The main purpose of this study is to explain how GBs deform at the atomic level, and to achieve this we first obtained their equilibrium structure. The GBs investigated in this study are listed in Table.1, and a detailed view of the $\mathrm{Cu}\langle 110>$ tilt GB structures with misorientation angles $0^{\circ}<\theta<180^{\circ}$ are show Fig.3 and Fig.4. Due to the symmetry of the fcc lattice, the misorientation angles from $0^{\circ}$ to $180^{\circ}$ covered all the distinct boundary structures of $\langle 110>$ tilt GBs. Snapshots of the atomic configuration at the GB area were taken using a molecular statics calculation by $0 \mathrm{~K}$ energy minimization procedure. The viewing direction was along the $\left[\begin{array}{lll}1 & \overline{1} & 0\end{array}\right]$ crystallographic direction $(\mathrm{Z}$ axis) and the positions of the atoms were projected onto the X-Y plane for clarity. The structural units proposed by Rittner and Seidman ${ }^{28}$ were used to illustrate the boundary structures. Atoms were shaded by their consecutive $\left(\begin{array}{lll}0 & 0 & 2\end{array}\right)$ atomic plane in order to identify the GB structural units.

Most boundary structures can generally be characterized by the structure unit model, as outlined by the solid line in Fig.3 and Fig.4, even though the GB structures were not symmetrical about the boundary plane. Of all the $\langle 110\rangle$ tilt GBs, three boundary structures were found to be special; $\sum 11\left(\begin{array}{lll}1 & 1 & 3\end{array}\right) \theta=50.5^{\circ} \mathrm{GB}, \sum 3\left(\begin{array}{lll}1 & 1 & 1\end{array}\right) \theta=109.5^{\circ} \mathrm{GB}$ and $\sum 9\left(\begin{array}{lll}2 & 2 & 1\end{array}\right) \theta=141.1^{\circ} \mathrm{GB}$, which was composed entirely of $\mathrm{C}, \mathrm{D}$, and $\mathrm{E}$ structural units respectively. These were considered to be the preferred structural unit model to represent GBs because other bicrystal boundaries with non-preferred misorientations consisted of two or more different types of preferred structural units. For example, the $\sum 9\left(\begin{array}{lll}1 & 1 & 4\end{array}\right)$ GB in Fig.3(a) contains two C and one D structural units per boundary period, the $\sum 11\left(\begin{array}{lll}3 & 3 & 2\end{array}\right)$ GB in Fig.4(b) contains one E and one D structural units per boundary period. It is worth noting that three boundaries in Fig.3(d)-(f) show a dissociated GB structure which was caused by the asymmetric dissociation of secondary GB dislocations with Burgers vector of the Shockley partial type ${ }^{28}$. This structure lowers the energy of the atomic arrangement in the boundary plane but also creates an extra stacking fault area inside the grain. To accommodate the intrinsic stacking fault facets, the $\mathrm{C}$ units were tilt downwards relative to the positive $\mathrm{X}$-axis, while the D unit lies at the termination of an intrinsic stacking fault that extended from the bicrystal interface.

Fig.4 lists six GB structures within a misorientation range of $\theta>109.5^{\circ}$, all of which contain the $\mathrm{E}$ structural units. 
Specifically, the GBs with $\theta<141.1^{\circ}$ contain a combination of $E$ and D units, while the GBs with $\theta>141.1^{\circ}$ contain a combination of $\mathrm{E}$ and $\mathrm{A}$ units. Notice that the $\mathrm{A}^{\prime}$ and $\mathrm{E}^{\prime}$ units marked in Fig.4(f) contain topologically identical atoms as $\mathrm{A}$ and $\mathrm{E}$ units, but they only differ in their direction. The $\left\langle\begin{array}{lll}1 & 0 & 0\end{array}\right.$ tilt GB structures presented here agree with the structures reported in the previous work ${ }^{28,37}$. Some of the GB structures obtained in this study using a molecular statics calculation for $\mathrm{Cu}$ are comparable with the images from a high resolution transmission electron microscopy (HRTEM) observation on other fcc metals $\left(\mathrm{Al}^{38,39}, \mathrm{Ni}^{40,41}\right.$ and $\left.\mathrm{Au}^{42}\right)$.

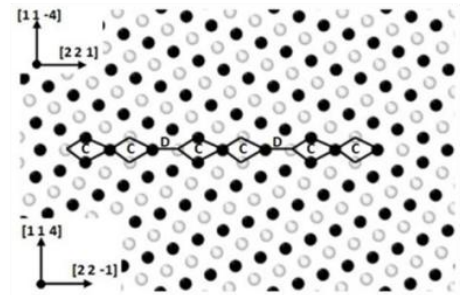

(a) $\sum 9$ (1 114$) \theta=38.9^{\circ}$

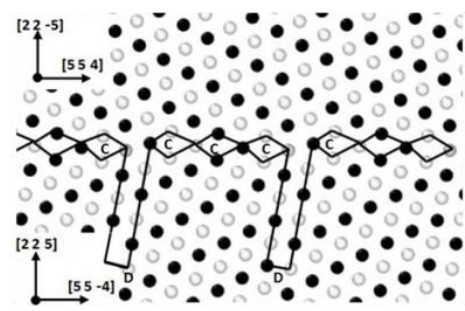

(d) $\sum 33(225) \theta=58.9$

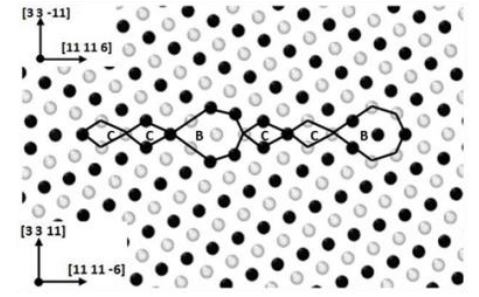

(b) $\sum 139$ (3 3 11) $\theta=42.2$

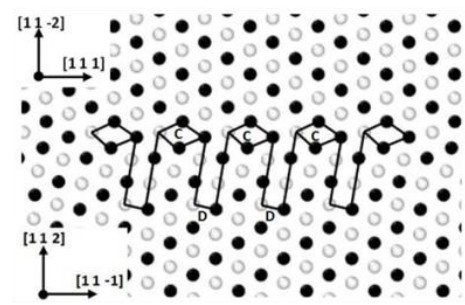

(e) $\sum 3\left(\begin{array}{lll}1 & 1 & 2\end{array}\right) \theta=70.5$

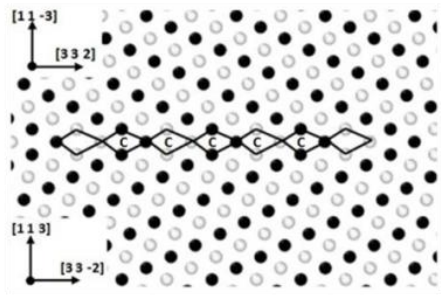

(c) $\Sigma 11\left(\begin{array}{llll}1 & 1 & 3\end{array}\right) \theta=50.5^{\circ}$

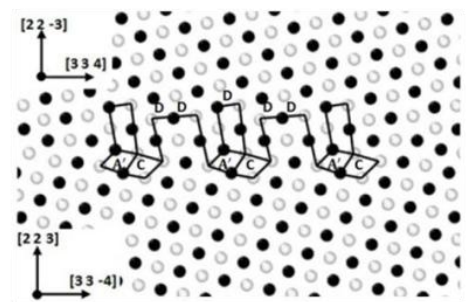

(f) $\sum 17$ (2 2 3) $\theta=86.6$

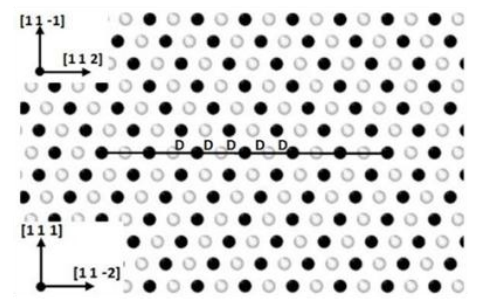

(g) $\sum 3\left(\begin{array}{lll}1 & 1 & 1\end{array}\right) \theta=109.5$

Figure-3. The equilibrium structures of the $\mathrm{Cu}<1 \quad 10>$ tilt GB structures with misorientation angles $\theta<109.5^{\circ}$ obtained by the energy minimization procedure and subsequent MD relaxation at $10 \mathrm{~K}$. The structures are viewed along the $\left[\begin{array}{lll}1 & \overline{1} & 0\end{array}\right]$ tilt axis. Atoms on consecutive $(00$ 2) planes are shown as black and white. The GB normal and period vectors are given for grain-A and grain-B on the left hand side of each structure. The structural units at each boundary plane are outlined by the solid line.

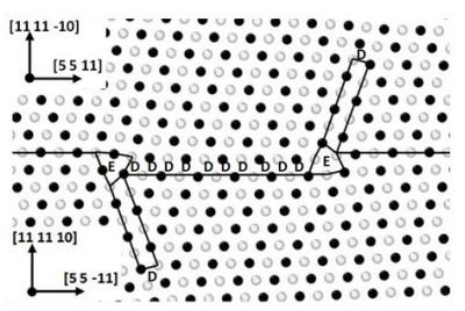

(a) $\Sigma 171$ (11 11 10) $\theta=114.5$

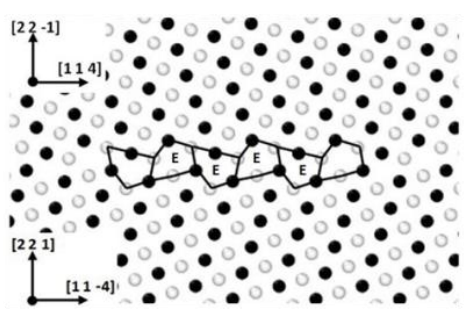

(d) $\sum 9$ (2 2 1) $\theta=141.1$

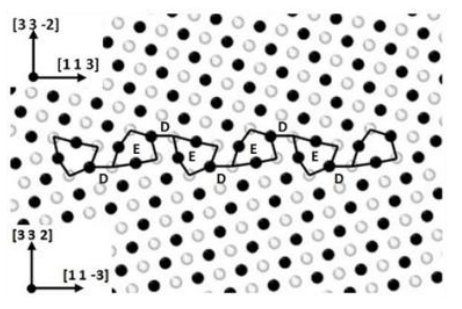

(b) $\sum 11$ (3 3 2 2) $\theta=129.5$

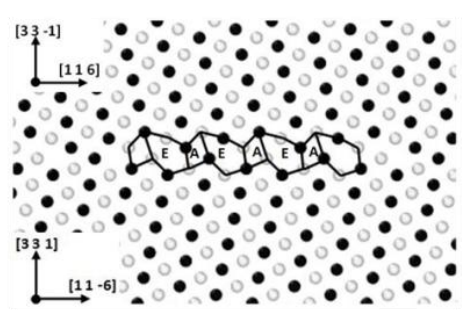

(e) $\sum 19$ (3 3 1 1) $\theta=153.5^{\circ}$

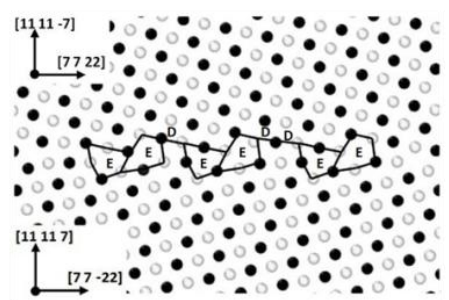

(c) $\sum 291$ (11 11 7) $\theta=131.5$

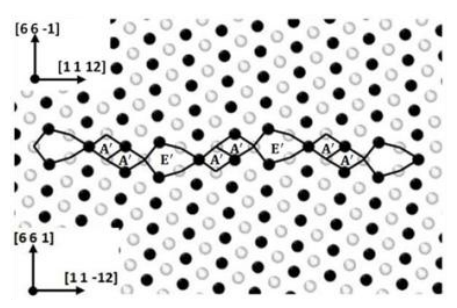

(f) $\Sigma 73(66$ 1) $\theta=166.6$

Figure-4. The equilibrium structures of the $\mathrm{Cu}<1 \quad 1 \quad 0>$ tilt GB structures with misorientation angles $\theta>109.5^{\circ}$ obtained by the energy minimization procedure and subsequent $\mathrm{MD}$ relaxation at $10 \mathrm{~K}$. 
The relationship of GB energy as a function of GB misorientation is plotted in Fig.5, and indicates that this curve contains local minimums and cusps corresponding to the two special GBs, $\sum 11\left(\begin{array}{lll}1 & 1 & 3\end{array}\right) \mathrm{GB}$ and $\sum 3\left(\begin{array}{lll}1 & 1 & 1\end{array}\right) \mathrm{GB}$, which are all composed entirely of the preferred structural units. This means that at a rotation of $0^{\circ}$ and $180^{\circ}$, the atoms are in perfect lattice configuration. At a rotation of $50.5^{\circ}$, the defect structure at the boundary plane is simple and therefore it corresponds to a local minimum energy. A $109.5^{\circ}$ tilt rotation about the $\langle 110\rangle$ axis has the lowest energy of any GB, and it corresponds to a very simple defect structure known as the coherent twin boundary.

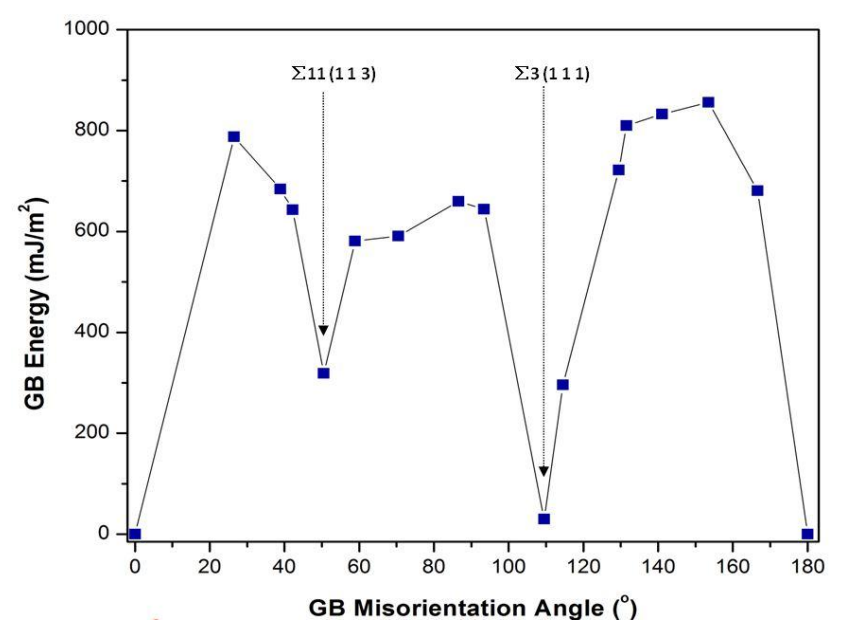

Figure-5. The energy of $\mathrm{Cu}<110>$ tilt GBs shown as a function of the misorientation angle at $0 \mathrm{~K}$ after energy minimization procedure.

\subsection{Tensile response}

After the equilibrium structure of GBs achieved, the simulation models were deformed under a uniaxial tensile loading at $10 \mathrm{~K}$. The stress-strain curves for the Cu bicrystal with different GBs are shown in Fig.6. The curves are separated as (a) and (b) corresponding to the GB structures with a misorientation angle where $\theta<109.5^{\circ}$ and $\theta>109.5^{\circ}$. The results showed a strong anisotropic of elastic modulus where the elastic stiffness of the bicrystal models increases as the misorientation angle increases within the range of $\theta<109.5^{\circ}$. This trend was opposite within the range of $\theta>109.5^{\circ}$. Moreover, the maximum tensile stress arrived at different strain rate for each GB and there was a big difference in the values; the calculated values are listed in Table.1.

The maximum tensile stress was plotted as a function of the GB misorientation angle and is shown in Fig.6(c). We also considered the role of lattice orientation on the maximum tensile stress, and have presented the calculations of single crystals with various orientation angles as a comparison. Here, the peak stress of $\mathrm{Cu}\langle 1 \quad 1 \quad 0\rangle$ single crystals generally increased as misorientation angle below $109.5^{\circ}$ increased, but then it gradually decreased as the misorientation angle increased above $109.5^{\circ}$. With the $\mathrm{Cu}$ bicrystals, the general trend of their peak stress was in accordance with $\mathrm{Cu}$ single crystals below $109.5^{\circ}$, although the overall values were lower than the single crystals and seemed to scatter more. The lower stress of the $\mathrm{Cu}$ bicrystal was due to the existence of GBs where the atoms have a higher energy than the lattice atoms and make the dislocation easier to nucleate. It was also interesting to find that the peak stress value of the bicrystals appeared to drop suddenly when the misorientation angle was greater than $109.5^{\circ}$, and the trend of peak stress within the range of $\theta>109.5^{\circ}$ deviated to the case of single crystals. This unique mechanical behavior of $\mathrm{Cu}$ bicrystals when $\theta>109.5^{\circ}$ can be attributed to their intrinsic GB structures with $\mathrm{E}$ structural units ${ }^{37,43,44}$, how the $\mathrm{E}$ units played a role as the deformation mechanism will be presented in the following discussion. Moreover, it was considered that the highest tensile strength of $\sum 3\left(\begin{array}{lll}1 & 1 & 1\end{array}\right)$ GB resulted from its simple boundary structure and lowest boundary energy. 

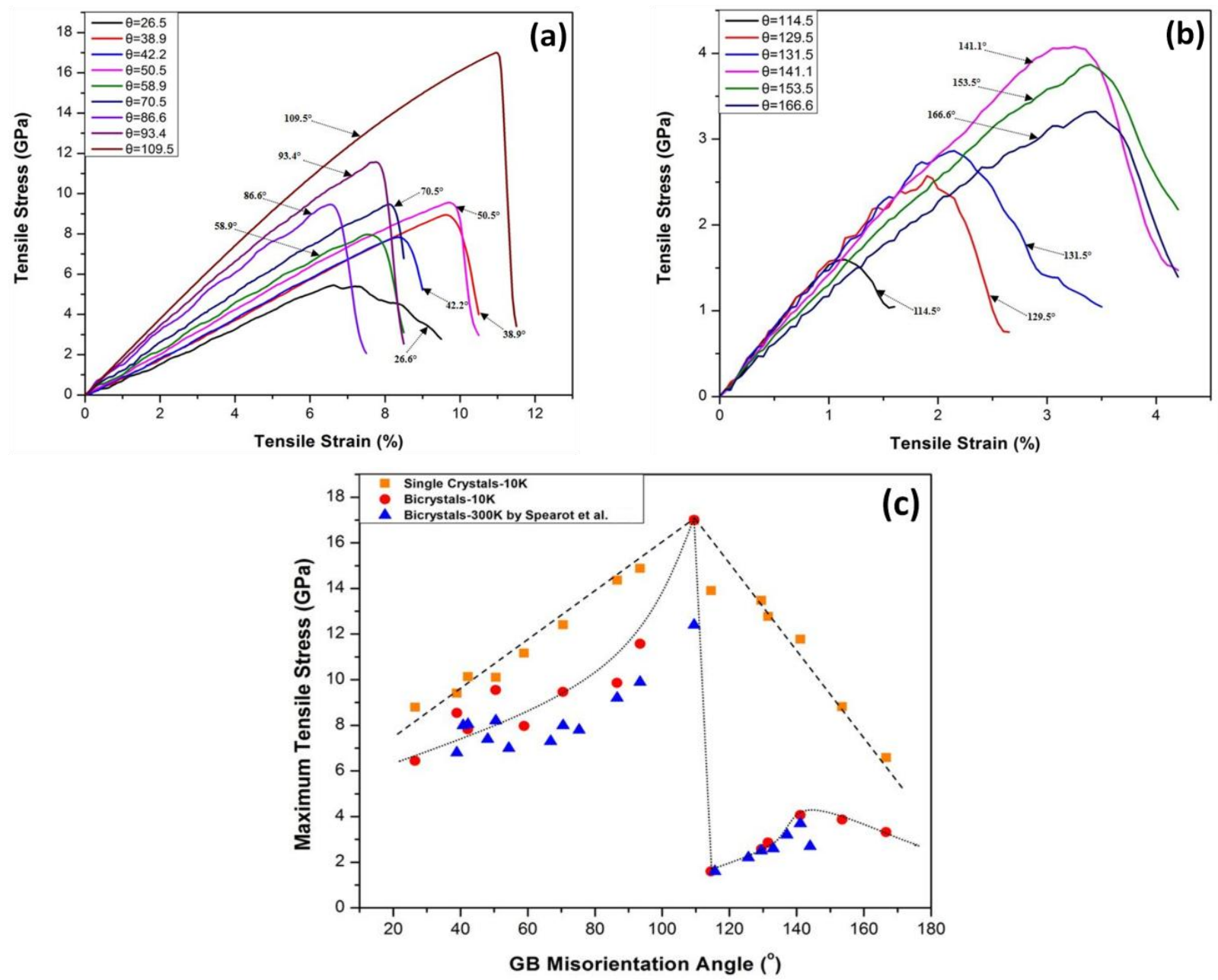

Figure-6. Stress-strain curves of $\mathrm{Cu}<110>$ tilt GBs with misorientation angles (a) $\theta<109.5^{\circ}$ and (b) $\theta>109.5^{\circ}$ at $10 \mathrm{~K}$. (c) Maximum tensile stress as a function of misorientation angle. The values of single crystals are plotted for comparison. The simulation results from Spearot $e t a l^{42}$ are also listed for comparison.

\subsection{Dislocation nucleation from $\mathrm{GB}$ with $\theta<109.5^{\circ}$}

During the early stage of loading, the system responds elastically and the lattices are stretched without dislocations formed. As the strain increases, a visual inspection of the MD simulation results indicated that the maximum tensile stress of $\mathrm{Cu}$ bicrystal models corresponded to the nucleation of dislocations from GBs. Fig.7(a)-(d) shows snapshots of four GBs with $\theta<109.5^{\circ}$ at the beginning of dislocation nucleation from the boundary plane at $10 \mathrm{~K}$. The images are colored according to the common neighbor analysis (CNA) parameter ${ }^{34}$. Only those atoms in defective arrangements are shown, the atoms with a perfect fcc environment were removed. The yellow atoms organized the GB plane and the dislocation core and the blue atoms represented the stacking fault. The corresponding energy barrier calculations for dislocations to nucleate from the GBs are shown in Fig.8(a)-(d). 


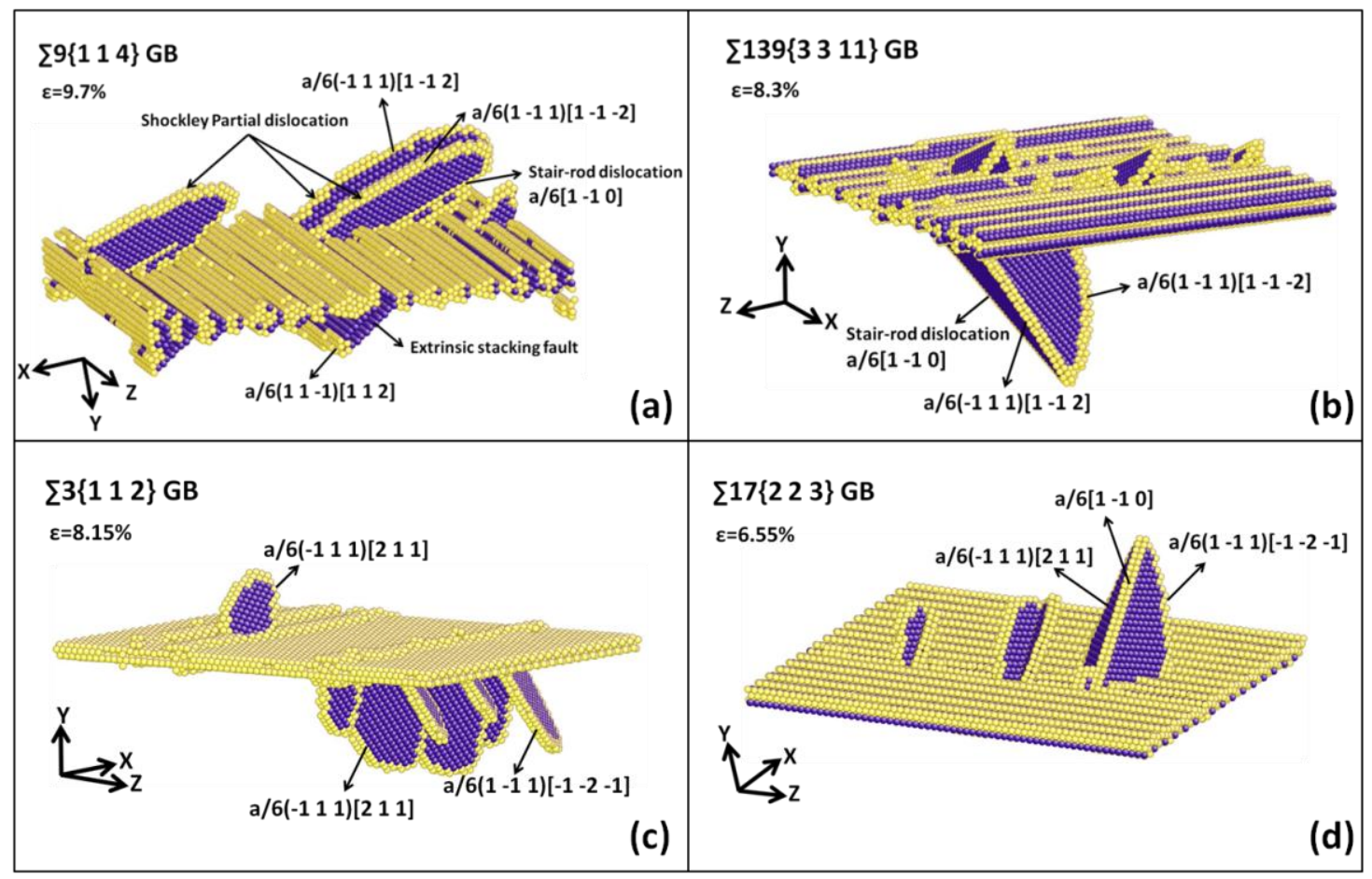

Figure-7. Dislocation nucleation from Cu bicrystal with (a) $\sum 9\left(\begin{array}{lll}1 & 1 & 4\end{array}\right) \theta=38.9^{\circ} \mathrm{GB}$ (b) $\sum 139\left(\begin{array}{llll}3 & 3 & 11\end{array}\right) \theta=42.2^{\circ} \mathrm{GB}$ (c) $\sum 3\left(\begin{array}{lll}1 & 1 & 2\end{array}\right) \theta=70.5^{\circ} \mathrm{GB}$ and (d) $\sum 17\left(\begin{array}{ll}2 & 2\end{array}\right) \theta=86.6^{\circ} \mathrm{GB}$ under uniaxial tension at $10 \mathrm{~K}$. Atoms with perfect fcc structures were removed to facilitate viewing the defective structures. The yellow atoms organized the GB plane and the dislocation core, and the blue atoms represented the stacking fault.
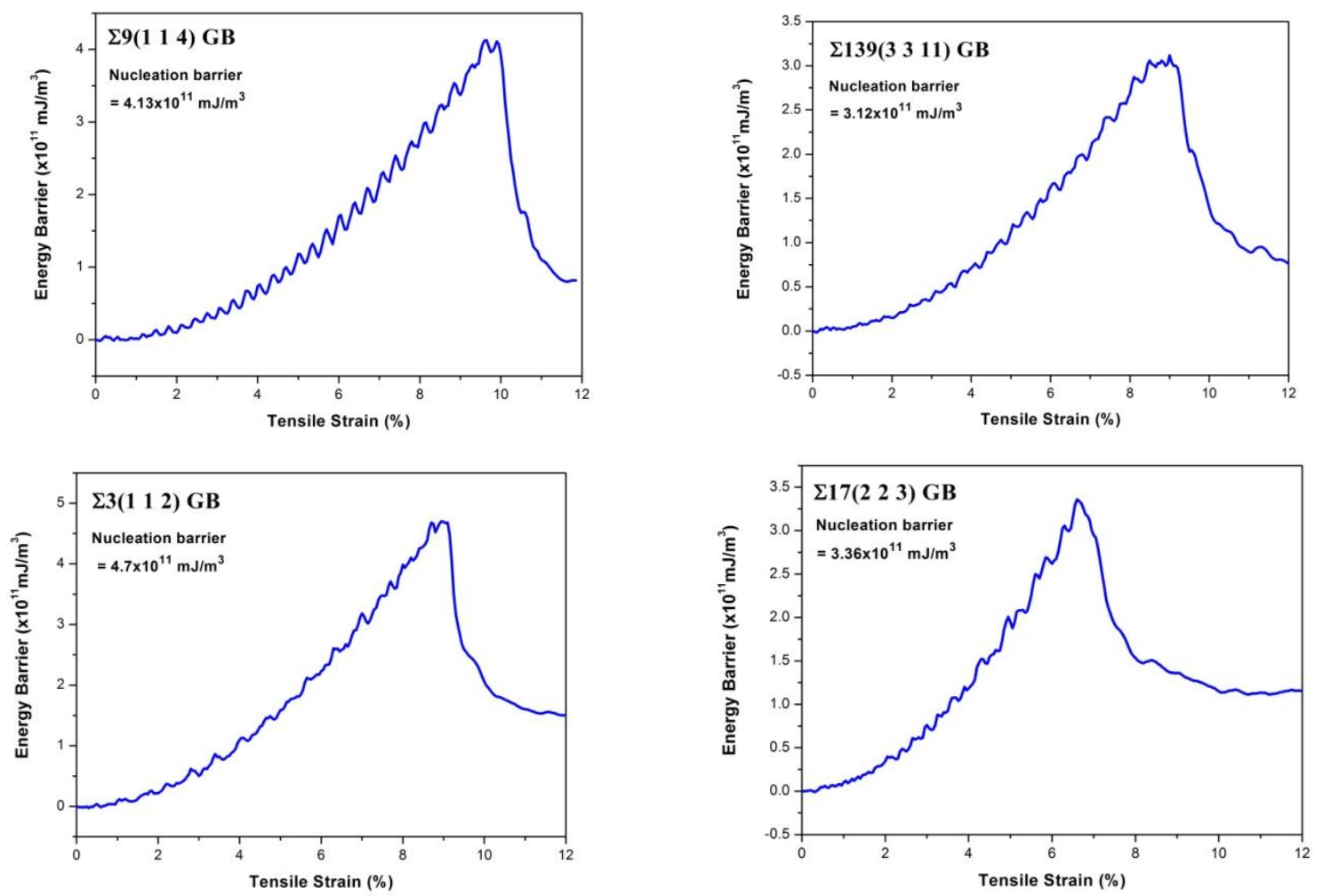

Figure-8. The calculated energy barrier for dislocations to nucleate from different GBs. (a) $\sum 9(114) \mathrm{GB}, \sum 139\left(3\right.$ 311) GB, $\sum 3(112) \mathrm{GB}$, and $\sum 17\left(\begin{array}{lll}2 & 2 & 3\end{array}\right) \mathrm{GB}$.

With the $\sum 9\left(\begin{array}{lll}1 & 1 & 4\end{array}\right) \mathrm{GB}$, partial dislocations were nucleated from the GB plane nearly simultaneously on both the primary and secondary slip systems, as shown with arrows in Fig.7(a). Some atoms on the GB plane became chaotic before they reached their maximum tensile stress, and eventually, the collective motion of the disordered atoms nucleated partial dislocation loops along the active slip planes within grain-B after reaching the peak stress at $\varepsilon=9.7 \%$. The nucleated 
dislocation loops had a V-shaped structure with an edge and screw dislocation character, where the exterior of the dislocation loop was the partial dislocation core that bound an intrinsic stacking fault. An examination of the V-shaped

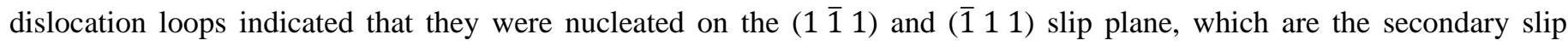
systems with a Schmid factor $\mathrm{SF}_{(1 \overline{1} 1)}=\mathrm{SF}_{(\overline{1} 11)}=0.419$ (see in Table.S1 for the calculation results of Schmid factor). The

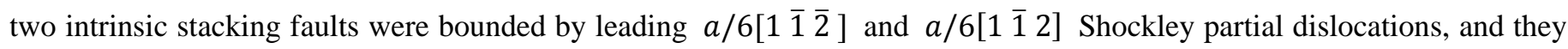

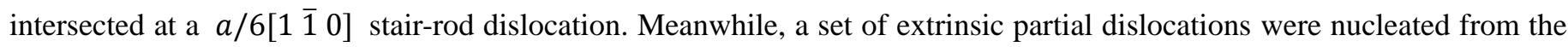
boundary plane into both grain-A and grain-B after the peak stress. This slip occurred on the $\left(\begin{array}{lll}1 & 1 & \overline{1}\end{array}\right)$ and (1 111$)$ planes respectively, which are the primary slip plane according to Schmid factor analysis with $\mathrm{SF}_{(11 \overline{1})}^{\max }=\mathrm{SF}_{(111)}^{\max }=0.471$. The nucleation of extrinsic partial dislocations resulted from the deformation of $\mathrm{C}$ structural units. This process will be fully illustrated in the following introduction of $\sum 11\left(\begin{array}{lll}1 & 1 & 3\end{array}\right) \mathrm{GB}$, which is composed entirely of C structural units. A further increase of the tensile strain caused dislocation interactions as the dislocation loops propagated throughout the crystal. As

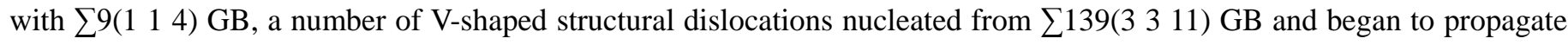
onto the $\left(\begin{array}{lll}1 & 1 & 1\end{array}\right)$ and $(\overline{1} 11)$ slip planes once the maximum tensile stress at $\varepsilon=8.3 \%$ had been reached, also the embryo of partial dislocations was evidenced on the (1 $1 \overline{1})$ and (1 11 ) planes, as shown in Fig.7(b).

The dislocation nucleation process of $\sum 11\left(\begin{array}{lll}1 & 1 & 3\end{array}\right)$ GB is presented in Fig.9. Recall that the $\sum 11\left(\begin{array}{lll}1 & 1 & 3\end{array}\right)$ GB had a simple boundary structure consisting of entirely $\mathrm{C}$ structural units, and the boundary energy was local minimum (the first cusp in Fig.5). The special boundary properties resulted in its different nucleation mechanisms. In Fig.9(a) at $\varepsilon=9.85 \%$, dislocations nucleated on the $\left(\begin{array}{lll}1 & 1 & \overline{1}\end{array}\right)$ and $\left(\begin{array}{lll}1 & 1 & 1\end{array}\right)$ plane from GB when the maximum tensile stress had been reached, and then they propagated symmetrically in grain-A and grain-B. They are the primary slip systems with $\mathrm{SF}_{(11 \overline{1})}^{\max }=\mathrm{SF}_{(111)}^{\max }=0.429$. Subsequently, the V-shaped partial dislocation loops began to nucleate in perfect lattice and slipped along the (1 $\overline{1} 1)$ and (1) 11 ) plane at $\varepsilon=10 \%$, as shown in Fig.9(b). The atoms that organized the $\sum 11\left(\begin{array}{lll}1 & 1 & 3\end{array}\right)$ GB were stable due to their comparative low boundary energy, so it was not as easy as the other cases where the V-shaped partial dislocation loops can nucleate from the boundary plane by shuffling local atoms. However, the intrinsic free volume of the $\mathrm{C}$ structural unit provided another path for the dislocations nucleating from the boundary along the secondary slip systems. This was seen at the sites of ' $a$ ' and ' $b$ ' in Fig.9(a), where an extrinsic stacking fault and a twin fault was generated after the dislocations nucleated, and more specifically, the distance of the twinning fault can become broad as the tensile strain increases, as the twin fault shows at 'b' in Fig.9(b).

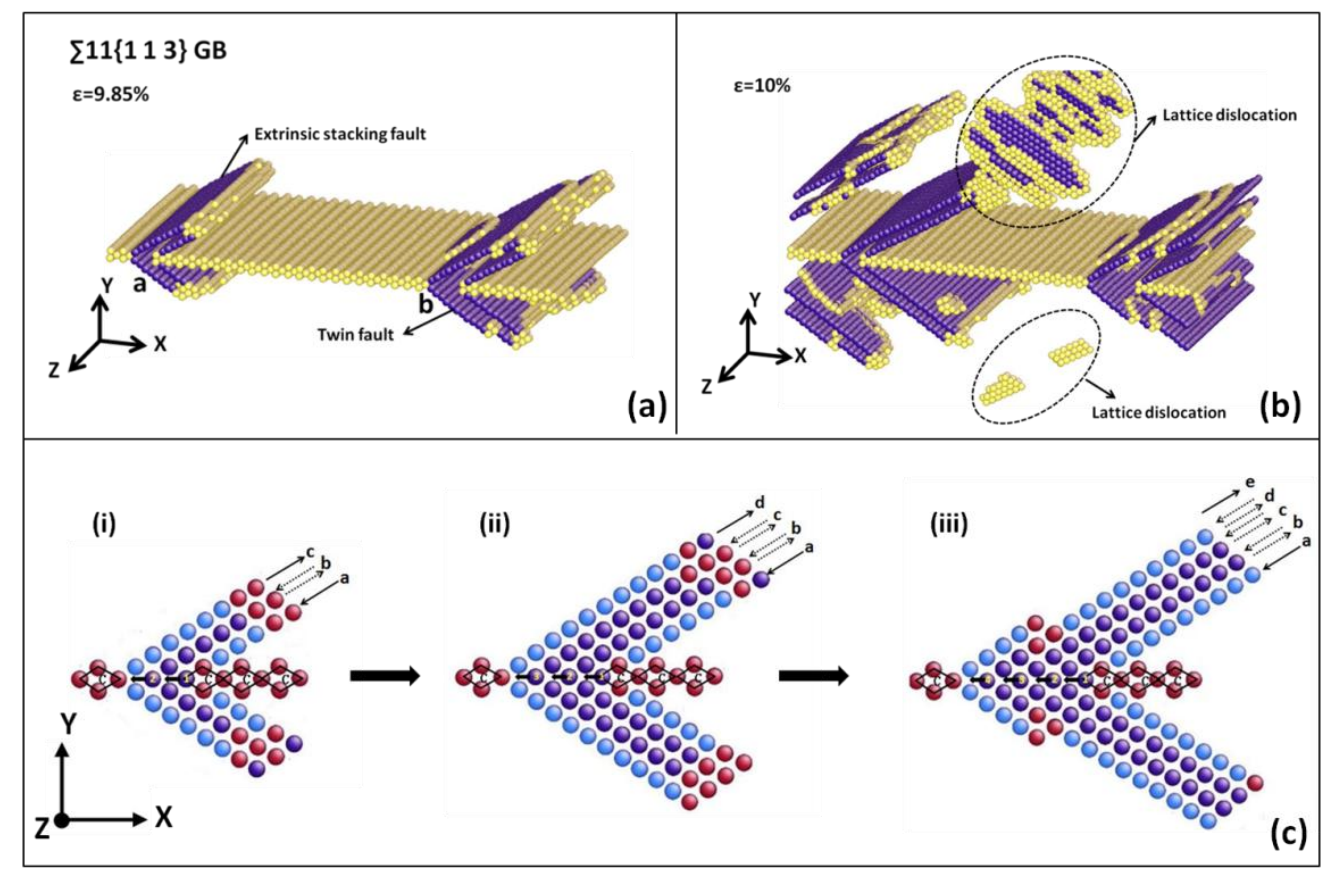

Figure-9. Dislocation nucleation from Cu bicrystal with $\sum 11\left(\begin{array}{lll}1 & 1 & 3\end{array}\right) \theta=50.5^{\circ} \mathrm{GB}$ under uniaxial tension at $10 \mathrm{~K}$. In Fig.(a) and (b), atoms with perfect fcc structures are removed and atoms of different colors represent the same as described in Fig.7. In Fig.(c), the dark blue atoms have the perfect fcc structure, atoms colored with red organize the GB plane and the dislocation core, the light blue atoms represent the twin fault. 
The nucleation process of an extrinsic stacking fault or a twin fault from $\sum 11\left(\begin{array}{lll}1 & 1 & 3\end{array}\right)$ GB is illustrated in Fig.9(c). On the whole, the uniaxial tensile strain caused the bicrystal to elongate in a $\mathrm{Y}$ direction and shortened the size of bicrystal in the $\mathrm{X}$ and $\mathrm{Z}$ directions. A careful examination of the images indicated that the bicrystal diminished in size in the $\mathrm{X}$ direction as a number of $\mathrm{C}$ structural units shrunk while driving the dislocation nucleation. Specifically, the first $\mathrm{C}$ unit shrank as a result of atom 1 translating in the negative $\mathrm{X}$ direction, which caused the atoms on plane ' $\mathrm{a}$ ' to slip towards the GB and the atoms on plane ' $b$ ' to slip out of the GB; this resulted in the first partial dislocation nucleation with an intrinsic stacking fault behind. Similarly, the translation of atom 2 caused the second partial dislocation nucleation by a relative shifting of atoms on plane ' $b$ ' and plane ' $c$ '. Notice that the slip direction caused by atom 2 on plane ' $b$ ' was opposite to that caused by atom 1, which drove the atoms on plane ' $b$ ' to turn back to the perfect fcc position and generated an extrinsic stacking fault, as shown in Fig.9(c)-(i). This mechanism of extrinsic stacking fault nucleation from GB through two partial dislocations emitted in adjacent compact planes was also evidenced in Fig.7(a) for $\sum 9\left(\begin{array}{lll}1 & 1 & 4\end{array}\right)$ GB. However, unlike $\sum 9\left(\begin{array}{lll}1 & 1 & 4\end{array}\right)$ GB, the consecutive shrinkage of $\mathrm{C}$ units along the boundary plane provided continuous nucleation sources for the consequent partial dislocations, and led to a broadening of the twinning region. As Fig.9(c)-(ii) and (iii) shows, the translation of atom 3 and atom 4 in the negative $\mathrm{X}$ direction resulted in the twin-boundary broadening to four and five (1 1111$)$ lattice spacing.

The GBs with a dissociated facet structures in the range of $50.5^{\circ}<\theta<109.5^{\circ}$ evolved before the dislocation nucleation event. Fig.7(c) and (d) show the $\sum 3\left(\begin{array}{lll}1 & 1 & 2\end{array}\right)$ GB and $\sum 17\left(\begin{array}{lll}2 & 2 & 3\end{array}\right)$ GB cases. The length of the dissociated intrinsic stacking fault decreased as the tensile deformation increased until it was accommodated entirely by the boundary plane.

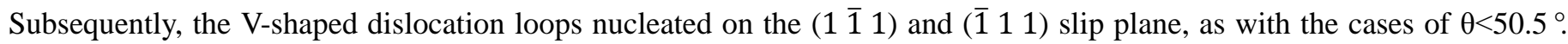
Notice that, according to Schmid factor analysis and the dislocation extraction algorithm (DXA) ${ }^{35,36}$, the $\left(\begin{array}{lll}1 & \overline{1} & 1\end{array}\right)$ and $\left(\begin{array}{lll}\overline{1} & 1 & 1\end{array}\right)$ slip plane are now the primary slip systems, and the Burgers vector of the two leading Shockley partial dislocations are

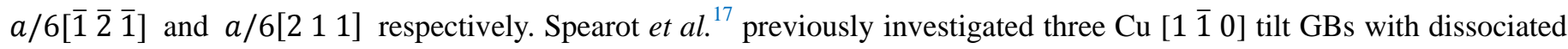
facet structures $\left(\theta=53.1^{\circ}, 53.1^{\circ}\right.$ and $\left.59^{\circ}\right)$. By comparing their findings and our simulation results, we conclude that as the misorientation angle of the GB is increased from $50.5^{\circ}$ to $109.5^{\circ}$, the spacing between intrinsic stacking fault facets decrease gradually, which causes the nucleation mechanisms change from one that is dominated by dislocation nucleation on secondary slip systems (i.e. slip on (1 $11 \overline{1})$ and (1 111$)$ planes) to that which is a mixture of dislocation nucleation on both primary and secondary slip systems, and finally change to one that is dominated by dislocation nucleation on primary slip systems (i.e. slip on ( $\left.\begin{array}{lll}1 & \overline{1} & 1\end{array}\right)$ and ( $(\overline{1} 11)$ planes).

\subsection{Dislocation nucleation with $\theta=109.5^{\circ}$ and $\theta=114.5^{\circ} \mathrm{GBs}$}

The maximum tensile stress of $\sum 3\left(\begin{array}{lll}1 & 1 & 1\end{array}\right) \mathrm{GB}$ was calculated as $17 \mathrm{GPa}$, which is the same as the maximum tensile stress of the corresponding single crystal with an orientation angle $\theta=109.5^{\circ}$. Recall that the $\sum 3\left(\begin{array}{lll}1 & 1 & 1\end{array}\right)$ coherent twin boundary had a very simple boundary structure and the lowest boundary energy of all the $\langle 110\rangle$ tilt GBs. This simple boundary structure has no excess free volume for local atoms to rearrange themselves at the boundary plane, and therefore it is hard to serve as a source of dislocation when the maximum tensile stress has been reached. Lattice dislocations nucleated homogeneously and their propagation intra-grains are visible in the bicrystal model, as shown in Fig.10(a). This is the same deformation mechanism as a single crystal, so the calculated tensile strength is identical. In Fig.10(b), the dislocation loops were

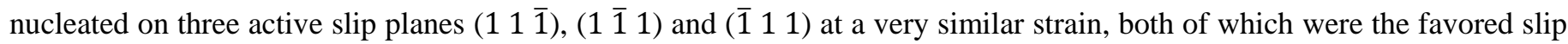
systems with the maximum Schmid factor $\mathrm{SF}_{(11 \overline{1})}^{\max }=\mathrm{SF}_{(1 \overline{1} 1)}^{\max }=\mathrm{SF}_{(1 \overline{1} \overline{1})}^{\max }=0.314$. No dislocation appeared to nucleate from the boundary plane during the process of tension deformation. Actually, the $\sum 3\left(\begin{array}{lll}1 & 1 & 1\end{array}\right) \mathrm{GB}$ is the only case in our study of the $\langle 110>$ tilt GBs that did not emit dislocations. 


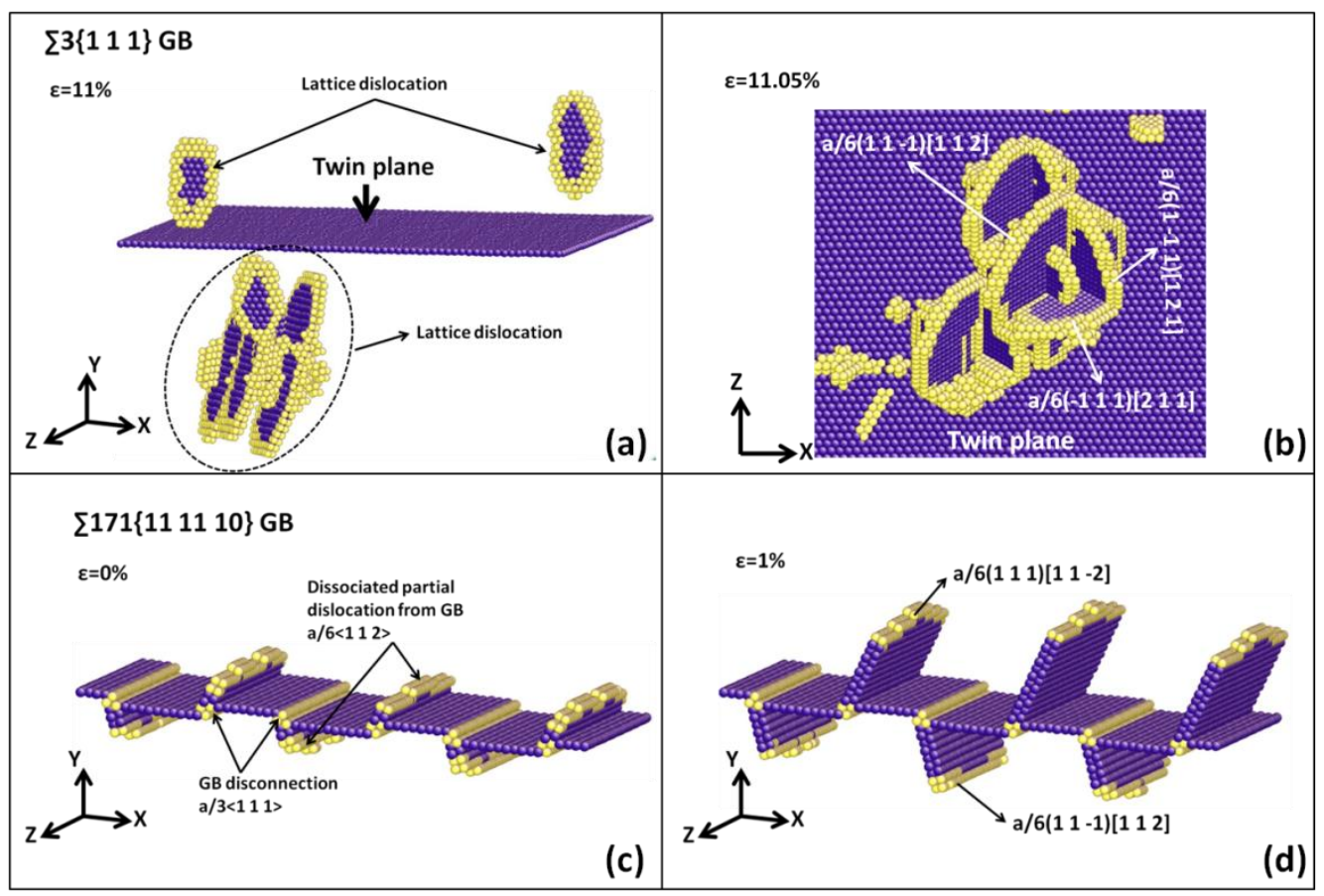

Figure-10. (a)-(b) Dislocation nucleation from Cu bicrystal with $\sum 3\left(\begin{array}{lll}1 & 1 & 1\end{array}\right) \theta=109.5^{\circ} \mathrm{GB}$ under uniaxial tension at $10 \mathrm{~K}$. (c)-(d) Dislocation nucleation from $\mathrm{Cu}$ bicrystal with $\sum 171\left(\begin{array}{lll}11 & 11 & 10\end{array}\right) \theta=114.5^{\circ} \mathrm{GB}$ under uniaxial tension at $10 \mathrm{~K}$. Atoms with perfect fcc structures are removed and atoms of different colors represent the same as described in Fig.7.

Compared to the $\sum 3\left(\begin{array}{lll}1 & 1 & 1\end{array}\right)$ coherent twin boundary, there was only a small deviation in lattice misorientation across the $\sum 171\left(\begin{array}{lll}11 & 11 & 10\end{array}\right) \theta=114.5^{\circ} \mathrm{GB}$. This kind of GB structure can be defined as a vicinal twin boundary ${ }^{45}$ where the $5^{\circ}$ misorientation is accommodated by a number of distorted $\mathrm{E}$ structural units and dissociated D structural units to provide a symmetrical structure. Following the work of Rittner and Merkle ${ }^{28}$, the distorted E units can be described as cores of alternating $\mathrm{a} / 3<111>$ twin dislocations, or disconnections, while the dissociated $\mathrm{D}$ units can be represented as a/6 $\langle 112\rangle$ Shockley partial dislocations. The a/3 $<111>$ disconnections were observed in previous experimental work by HRTEM in some fcc metals ${ }^{42,46,47}$. The $\sum 171\left(\begin{array}{lll}11 & 11 & 10\end{array}\right)$ GB disconnection and its dissociated partial dislocations are indicated by the arrows in Fig.10(c), and a detailed view can be seen in Fig.4(a). It is worth noting that, unlike the structure of GBs with $50.5^{\circ}<\theta<109.5^{\circ}$, the dissociated facet in $\sum 171(111110) \mathrm{GB}$ was along the primary slip plane in grain-A and Grain- $\mathrm{B}$, and therefore when subjected to the uniaxial tensile deformation, the pre-nucleated Shockley partial dislocations can propagate rapidly with the increasing length of intrinsic stacking fault behind at $\varepsilon=1.4 \%$, as shown in Fig.10(d). Since the partial dislocations have already nucleated in the equilibrium boundary structure, only a low stress can drive it to emit, which can explain the sharp decrease in the peak stress from the $\sum 3\left(\begin{array}{lll}1 & 1 & 1\end{array}\right)$ coherent twin boundary to the $\sum 171\left(\begin{array}{llll}11 & 11 & 10\end{array}\right)$ vicinal twin boundary.

\subsection{Dislocation nucleation from $\mathrm{GB}$ with $\theta>114.5^{\circ}$}

As was introduced previously, all the GBs with a misorientation angle of $\theta>109.5^{\circ}$ contained the $\mathrm{E}$ structural units. Sansoz and Molinari ${ }^{48,49}$ correlated the E structural unit with the incidence of atomic shuffling during shear deformation and proposed that the free volume inherent to this structural feature triggered the atomic shuffling event, while Spearot $e t$ $a l .^{37,50}$ correlated the mechanical behavior of $\mathrm{Cu}<1 \quad 10>$ GBs with the intrinsic large free volume of the $\mathrm{E}$ structural unit and its evolution during the dislocation nucleation. How the $\mathrm{E}$ units acted as the source of dislocation nucleation in different $\left\langle 110>\right.$ GBs with $\theta>114.5^{\circ}$ has been investigated in this study.

Again, a visual inspection of the MD simulation results indicated that the maximum tensile stress corresponded to the nucleation of partial dislocations from GBs in the range of $\theta>109.5^{\circ}$. Fig.11 shows the images of dislocation nucleation

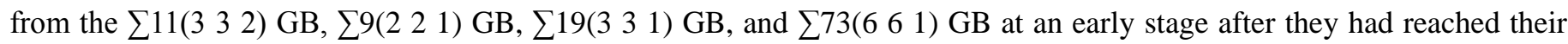
maximum tensile stress, and the corresponding energy barrier calculations for dislocations to nucleate from the GBs are shown in Fig.12(a)-(d). Fig.11 shows that the Shockley partial dislocations have nucleated and propagated along (1 111$)$ and 
(1 1 1 $\overline{1}$ ) plane, after originating from the collapsed E units that were connected to the boundary by a stacking fault. They are the primary slip systems according to the Schmid analysis. Notice here that the nucleation of Shockley partial dislocations were not nucleated collectively in all the $\mathrm{E}$ units from the boundary plane and only certain nucleated dislocations propagated away from the plane. Interestingly, the collapsed E units evolved into C structural units shown in Fig.11(a), (b) and (d), Where in Fig.11(c), the collapsed E units did not evolve into the C units like the other three GBs, which indicated there was a different deformation mechanism. To reduce the article body, description of the deformation process was provided in the supplementary document. Fig.S1 shows a detailed view of the transformation of the E structural units in different GBs during the dislocation nucleation process where one structural period of each GB was extracted for analysis.

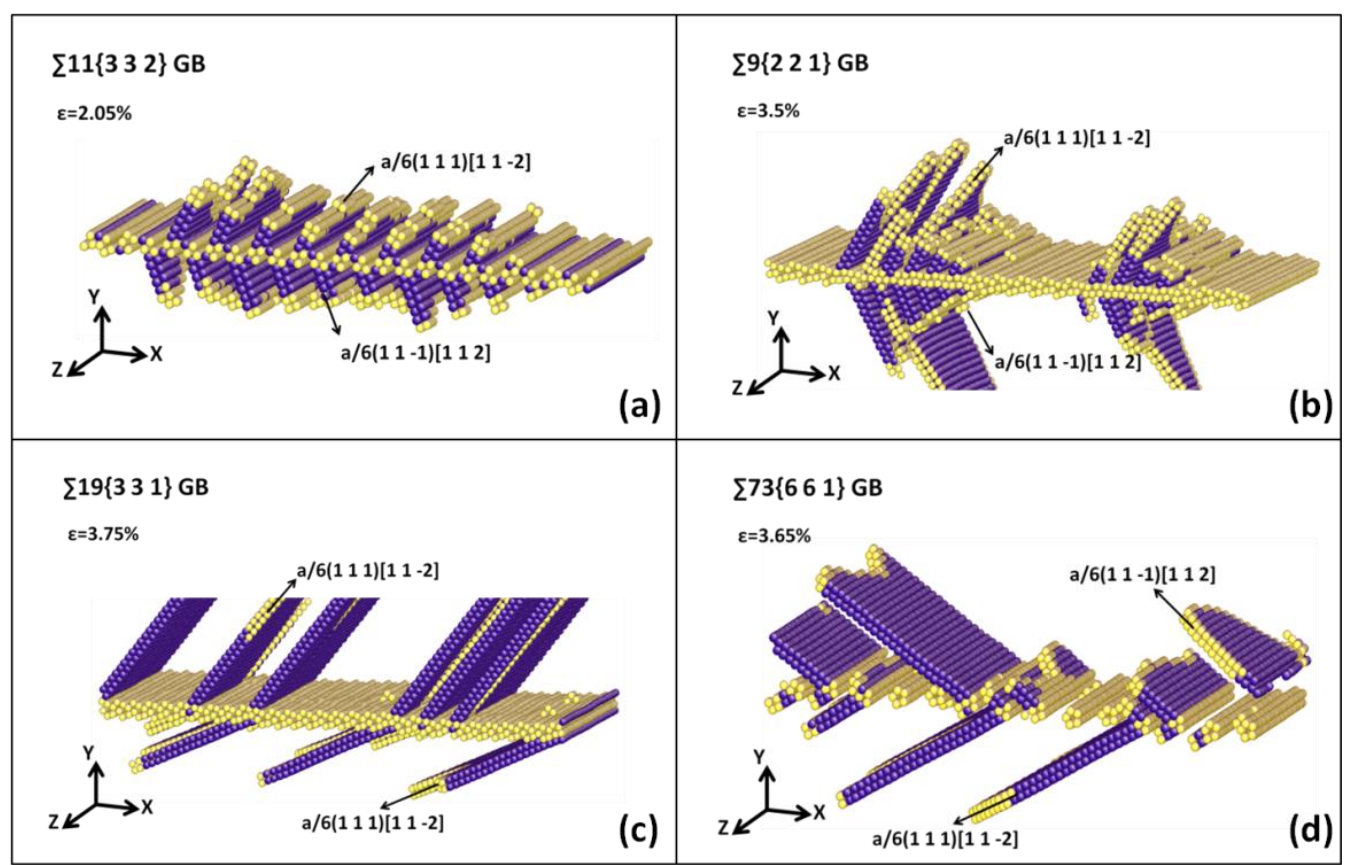

Figure-11. Dislocation nucleation and propagation from (a) $\sum 11$ (3 3 2) GB, (b) $\sum 9\left(22\right.$ 1) GB, (c) $\sum 19\left(\begin{array}{l}3 \\ 3\end{array}\right.$ 1) GB and (d) $\sum 73$ (6 6 1) GB under a uniaxial tension at $10 \mathrm{~K}$. Atoms with perfect fcc structures are removed and atoms of different colors represent the same as described in Fig.7.
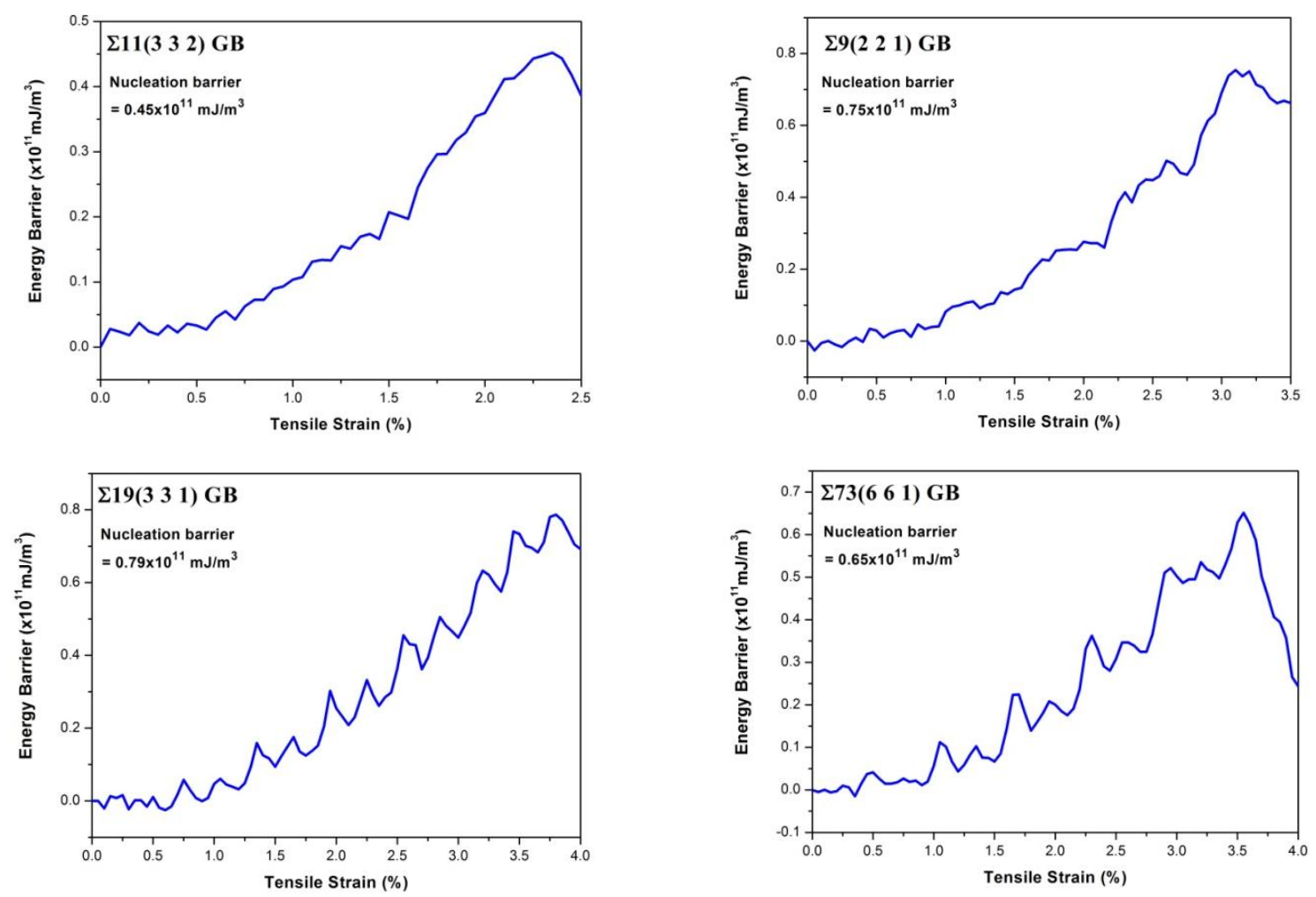

Figure-12. The calculated energy barrier for dislocations to nucleate from different GBs. (a) $\sum 11(332) \mathrm{GB}, \sum 9(221) \mathrm{GB}, \sum 19(33$ 1) GB, and 


\section{Remarks and conclusions}

In every case of this study, the maximum tensile stress of the bicrystal models corresponded to the heterogeneous nucleation of partial dislocations from GBs, except for the $\sum 3\left(\begin{array}{lll}1 & 1 & 1\end{array}\right) \theta=109.5^{\circ}$ coherent twin boundary due to its simple and stable boundary structure and extremely low boundary energy. Within the misorientation range of $\theta<109.5^{\circ}$, the tensile strength of bicrystals showed a consistent trend with the value of single crystals, which implied that lattice crystallographic geometry played a dominant role on the GB mechanical behavior. The incipient plastic deformation of bicrystal models was evidenced by a number of V-shaped partial dislocation loops nucleated from GB plane, as well as the nucleation of extrinsic stacking fault or twin fault. The maximum tensile stress of GBs with $\theta>109.5^{\circ}$ was much lower than the value of single crystals and showed a different trend. The simulation results indicated that the intrinsic GB structure, instead of having a lattice orientation, became the predominant factor that determined the mechanical behavior of GBs in this range.

The maximum tensile stress of each GB was plotted in Fig.13 as a function of GB energy. The energy barrier of dislocation nucleation from different GBs against their maximum tensile stress was plotted in Fig.14. Since the $\sum 3\left(\begin{array}{lll}1 & 1 & 1\end{array}\right)$ GB (coherent twin boundary) has a very simple structure and a stable configuration, the dislocations were nucleated in the matrix lattice without any dislocation nucleation from the GB plane during the simulation, which prevented the result of energy barrier calculation in Fig.14. There is generally an inverse relationship between the GB tensile strength and GB energy, while the energy barriers associated with dislocation nucleation for GBs showed a positive relationship to the tensile strength. These results indicated that the lower energy GBs with more stable boundary structures required higher tensile stress to nucleate dislocations during the onset of plastic deformation, and therefore the nucleation barrier was correspondingly higher. This was the case for GBs with $\theta \leq 109.5^{\circ}$. In reverse, for the less stable GBs $\left(\theta>109.5^{\circ}\right)$ with a higher boundary energy, a lower tensile stress can overcome the energy barrier to activate the dislocation nucleation from GBs. However, GB energy alone cannot determine the GB tensile strength and its nucleation barrier. For example, the energy of $\sum 11\left(\begin{array}{lll}1 & 1 & 3\end{array}\right) \theta=50.5^{\circ}$ GB corresponds to the local energy cusp in Fig.3, but its tensile strength (9.55 GPa) and the nucleation barrier $\left(4.85 \mathrm{~mJ} / \mathrm{m}^{3}\right)$ were not the local maximum, and in addition, there appears to be no explicit relationship between GB energy and the maximum tensile stress in the two circled areas shown in Fig.13.

Based on results of this investigation, the geometry of the bicrystal system or the orientation of the applied loading played an important role in the process of dislocation nucleation because the single crystal calculations showed a consistent trend with the results of bicrystal GBs in the range of $\theta<109.5^{\circ}$ shown in Fig.6(c). But it failed to explain the abrupt drop in tensile stress in the range of $\theta>109.5^{\circ}$, where the GB structure was determined to play a dominant role. The intrinsic large free volume (E structural units) involved in the boundary plane of GBs with $\theta>109.5^{\circ}$ resulted in a high boundary energy and provided enough space for the GB atoms to be rearranged during tension deformation to facilitate the dislocation nucleation and accommodate the local stress. In addition, the impact of GB structure on dislocation nucleation was also evidenced by GBs with C structural units. For example, the consecutive shrinkage of C units along the $\sum 11\left(\begin{array}{lll}3 & 3 & 2\end{array}\right)$ GB provided a nucleation source for an extrinsic stacking fault and a twin fault. Moreover, a very special case was found for the $\sum 171\left(\begin{array}{lll}11 & 11 & 10\end{array}\right) \mathrm{GB}$ (vicinal twin boundary). It had the second lowest boundary energy $\left(296 \mathrm{~mJ} / \mathrm{m}^{2}\right)$ of all the GBs investigated in this study, but its tensile strength $(1.6 \mathrm{GPa})$ and nucleation barrier $\left(0.1 \mathrm{~mJ} / \mathrm{m}^{3}\right)$ was the lowest. This contradicted the general trend shown in Fig.13, where the lower boundary energy caused higher nucleation stress. As mentioned previously, this was mainly due to the dissociated dislocation from the boundary plane in the equilibrium structure of $\sum$ 171(11 11 10) GB which made the nucleation process become very easy. This result further emphasizes the important role that GB structures play in the mechanical property of materials. 


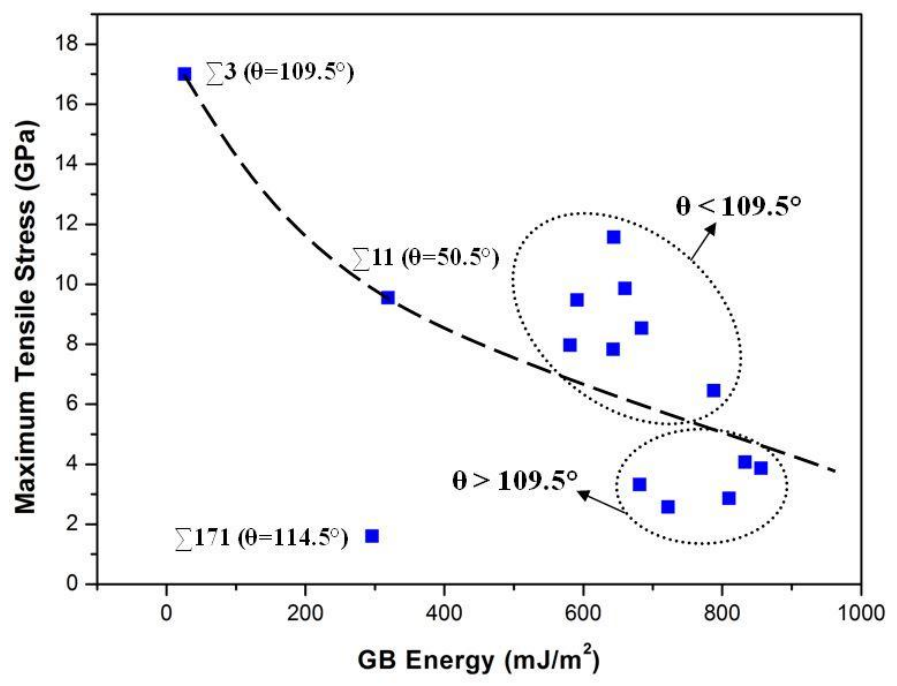

Figure-13. Maximum tensile stress of GBs plotted as a function of GB energy.

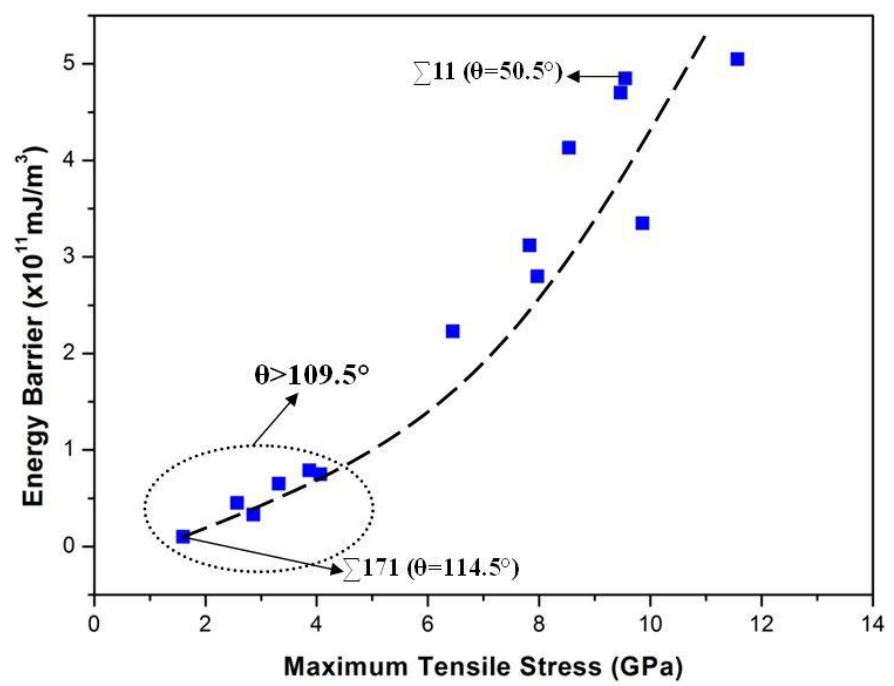

Figure-14. Energy barrier of dislocation nucleation from GB plotted as a function of maximum tensile stress.

In conclusion, molecular dynamics simulations were conducted on Cu bicrystals with different $\langle 110\rangle$ tilt GBs to study their structures, energy, mechanical property, and the dislocation nucleation mechanisms under tensile loading. In this study, some of the GB properties were quantified, these included GB energy, GB tensile strength, and the dislocation nucleation barrier on GB. We presented the atomistic mechanisms of the dislocation nucleation from various GBs and investigated their correlation with GB properties. The results of this study can help us to better understand the dislocation source in GB, and provide a theoretical basis for grain boundary engineering (GBE) to design the GB character distribution to attain certain bulk polycrystalline properties.

\section{Acknowledgements}

This work is supported by Australia Research Council Discovery Project (DP130103973). Simulations were performed using the HPC cluster of University of Wollongong and the computing facilities provided by NCI National Facility of Australia. L.Z., L.P and X.Z would like to acknowledge the financial support from China Scholarship Council (CSC).

\section{References}

1. Van Swygenhoven, H. Science 296, 66-67 (2002).

2. Fougere, G.E., Weertman, J.R., Siegel, R.W. \& Kim, S. Scr. Mater. 26, 1879-1883 (1992).

3. Scattergood, R.O. \& Koch, C.C. Scr. Mater. 27, 1195-1200 (1992).

4. Wang, Y.M., Hamza, A.V. \& Ma, E. Acta Mater. 54, 2715-2726 (2006).

5. Champion, Y. et al. Science 300, 310-311 (2003). 
6. Chen, M. et al. Science 300, 1275-1277 (2003).

7. Shan, Z. et al. Science 305, 654-657 (2004).

8. Yamakov, V., Wolf, D., Phillpot, S.R., Mukherjee, A.K. \& Gleiter, H. Nature Mater. 1, $45-48$ (2002).

9. Yamakov, V., Wolf, D., Phillpot, S.R. \& Gleiter, H. Acta Mater. 51, 4135-4147 (2003).

10. Van Swygenhoven, H. \& Weertman, J.R. Mater. Today 9, 24-31 (2006).

11. Schiøtz, J., Vegge, T., Di Tolla, F.D. \& Jacobsen, K.W. Phys. Rev. B 60, 11971-11983 (1999).

12. Schiøtz, J. Scr. Mater. 51, 837-841 (2004).

13. Mishin, Y., Asta, M. \& Li, J. Acta Mater. 58, 1117-1151 (2010).

14. Van Swygenhoven, H., Derlet, P.M. \& Frøseth, A.G. Acta Mater. 54, 1975-1983 (2006).

15. Van Swygenhoven, H., Derlet, P.M. \& Hasnaoui, A. Phys. Rev. B 66, 241011-241018 (2002).

16. Spearot, D.E., Jacob, K.I. \& McDowell, D.L. Acta Mater. 53, 3579-3589 (2005).

17. Spearot, D.E., Jacob, K.I. \& McDowell, D.L. Int. J. Plast. 23, 143-160 (2007).

18. Tschopp, M.A. \& McDowell, D.L. Int. J. Plast. 24, 191-217 (2008).

19. Zhang, L., Lu, C. \& Tieu, K. Sci. Rep. 4, 5919 (2014).

20. Liao, X.Z. et al. Appl.Phys.Lett. 83, 632-634 (2003).

21. Liao, X.Z. et al. Appl.Phys.Lett. 84, 3564-3566 (2004).

22. Van Swygenhoven, H. \& Derlet, P.M. Dislocations in Solids. 14, 1-42 (2008).

23. Tschopp, M.A., Spearot, D.E. \& McDowell, D.L. Dislocations in Solids. 14, 43-139 (2008).

24. Tucker, G.J. \& McDowell, D.L. Int. J. Plast. 27, 841-857 (2011).

25. Randle, V. Acta Mater. 46, 1459-1480 (1998).

26. Plimpton, S. J. Comput. Phys. 117, 1-19 (1995).

27. Mishin, Y., Mehl, M.J., Papaconstantopoulos, D.A., Voter, A.F. \& Kress, J.D. Phys. Rev. B 63, 2241061-22410616 (2001).

28. Rittner, J.D. \& Seidman, D.N. Phys. Rev. B 54, 6999-7015 (1996).

29. Olmsted, D.L., Foiles, S.M. \& Holm, E.A. Acta Mater. 57, 3694-3703 (2009).

30. Zhang, L., Lu, C., Michal, G., Tieu, K. \& Cheng, K. Mater. Res. Express 1, 015019 (2014).

31. Sangid, M.D., Ezaz, T., Sehitoglu, H. \& Robertson, I.M. Acta Mater. 59, 283-296 (2011).

32. Li, J. Model. Simul. Mater. Sci. Eng. 11, 173-177 (2003).

33. Stukowski, A. Model. Simul. Mater. Sci. Eng. 18, 015017 (2010).

34. Schiøtz, J., Di Tolla, F.D. \& Jacobsen, K.W. Nature 391, 561-563 (1998).

35. Stukowski, A. Model. Simul. Mater. Sci. Eng. 20, 045021 (2012).

36. Stukowski, A. \& Albe, K. Model. Simul. Mater. Sci. Eng. 18, 085001 (2010).

37. Tschopp, M.A., Tucker, G.J. \& McDowell, D.L. Acta Mater. 55, 3959-3969 (2007).

38. Medlin, D.L., Mills, M.J., Stobbs, W.M., Daw, M.S. \& Cosandey, F. Mater. Res. Soc. Symp. Proc. 295, 91-96 (1993).

39. Mills, M.J., Daw, M.S., Thomas, G.J. \& Cosandey, F. Ultramicroscopy 40, 247-257 (1992).

40. Hardouin Duparc, O., Poulat, S., Larere, A., Thibault, J. \& Priester, L. Mater. Sci. Eng. A 80, 853-870 (2000).

41. Poulat, S., Thibault, J. \& Priester, L. Interface Sci. 8, 5-15 (2000).

42. Marquis, E.A. \& Medlin, D.L. Philos. Mag. Lett. 85, 387-394 (2005).

43. Tucker, G.J., Tschopp, M.A. \& McDowell, D.L. Acta Mater. 58, 6464-6473 (2010).

44. Spearot, D.E., Tschopp, M.A., Jacob, K.I. \& McDowell, D.L. Acta Mater. 55, 705-714 (2007).

45. Frøseth, A.G., Derlet, P.M. \& Van Swygenhoven, H. Scripta Mater. 54, 477-481 (2006).

46. Medlin, D.L., Carter, C.B., Angelo, J.E. \& Mills, M.J. Philos. Mag. A 75, 733-747 (1997).

47. Foiles, S.M. \& Medlin, D.L. Mater. Sci. Eng. A 319, 102-106 (2001).

48. Sansoz, F. \& Molinari, J.F. Scr. Mater. 50, 1283-1288 (2004).

49. Sansoz, F. \& Molinari, J.F. Acta Mater. 53, 1931-1944 (2005).

50. Spearot, D.E. Mech. Res. Comm. 35, 81-88 (2008). 